EPJ Web of Conferences 66, 01004 (2014)

DOI: $10.1051 /$ epjconf/ 20146601004

(C) Owned by the authors, published by EDP Sciences, 2014

\title{
Meson Spectroscopy in the Light Quark Sector
}

\author{
R. De Vita ${ }^{1, a}$ \\ ${ }^{1}$ Istituto Nazionale di Fisica Nucleare, Sezione di Genova, Via Dodecaneso 33, 16146 Genova, Italy
}

\begin{abstract}
Understanding the hadron spectrum is one of the fundamental issues in modern particle physics. We know that existing hadron configurations include baryons, made of three quarks, and mesons, made of quark-antiquark pairs. However most of the mass of the hadrons is not due to the mass of these elementary constituents but to their binding force. Studying the hadron spectrum is therefore a tool to understand one of the fundamental forces in nature, the strong force, and Quantum Chromo Dynamics (QCD), the theory that describes it. This investigation can provide an answer to fundamental questions as what is the origin of the mass of hadrons, what is the origin of quark confinement, what are the relevant degrees of freedom to describe these complex systems and how the transition between the elementary constituents, quarks and gluons, and baryons and mesons occurs.

In this field a key tool is given by meson spectroscopy. Mesons, being made by a quark and an anti-quark, are the simplest quark bound system and therefore the ideal benchmark to study the interaction between quarks and understand what the role of gluons is. In this investigation, it is fundamental to precisely determine the spectrum and properties of mesons but also to search for possible unconventional states beyond the $q \bar{q}$ configuration as tetraquarks $(q q \bar{q} \bar{q})$, hybrids $(q \bar{q} g)$ and glueballs. These states can be distinguished unambiguously from regular mesons when they have exotic quantum numbers, i.e. combinations of total angular momentum, spin and parity that are not allowed for $q \bar{q}$ states. These are called exotic quantum numbers and the corresponding states are referred to as exotics.

The study of the meson spectrum and the search for exotics is among the goals of several experiments in the world that exploit different reaction processes, as $e^{+} e^{-}$annihilation, $p \bar{p}$ annihilation, pion scattering, proton-proton scattering and photo-production, to produce meson states. This intense effort is leading to a very rich phenomenology in this sector and, together with recent theoretical progress achieved with lattice QCD calculations, is providing crucial information to reach a deeper understanding of strong interaction.

In these proceedings I will review the present status of meson spectroscopy in the light quark sector and the plans and perspectives for future experiments.
\end{abstract}

\section{Introduction}

Since ancient times, understanding the structure of matter around us has been one of the main objectives of science. Nowadays, we know that most of the visible mass of the universe is due to hadrons and, in particular, to the protons and neutrons that constitute the atomic nucleus. We also know that

ae-mail: raffaella.devita@ge.infn.it

This is an Open Access article distributed under the terms of the Creative Commons Attribution License 2.0, which permits unrestricted use, distribution, and reproduction in any medium, provided the original work is properly cited. 

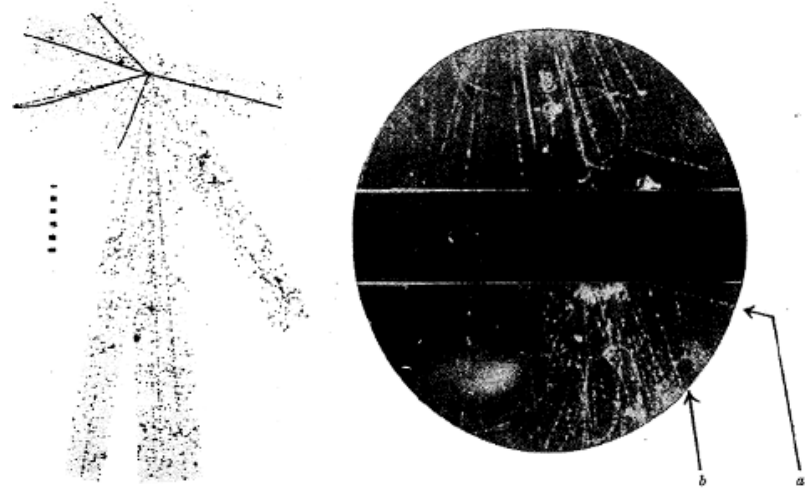

Figure 1. Left: photographic emulsion image of a cosmic particle disintegration into pions [1]. Right: Cloud chamber photograph showing a forked track decay of a $K^{0}$ into $\pi^{+} \pi^{-}$observed by Rochester and Butler [2].

hadrons are complex systems, being made of quarks interacting via the exchange of gluons. Known quarks configurations are baryons, made of three quarks, and mesons, made of quark-antiquark pairs. While this picture of hadronic matter seems now well established and clear, many open questions and puzzles still remain. Among these, one of the most surprising fact is that only a small fraction of the hadron mass seems to be associated to the mass of the elementary quarks. In fact, the combined mass of the uud quarks forming the proton is of the order of $10 \mathrm{MeV}$ and therefore accounts only for $\sim 1 \%$ of the proton mass. It is now understood that the remaining fraction of the mass is due to the force that binds the quarks within hadrons, i.e. Quantum Chromo Dynamics (QCD). We can therefore say that is QCD with its rules and constraints that determines the mass and spectrum of hadrons and makes the world around us as we know it.

Studying the structure and properties of hadrons is therefore crucial to reach a deep understanding of QCD and answer fundamental questions such as as what is the origin of the mass of hadrons, what is the origin of quark confinement, what are the relevant degrees of freedom to describe these complex systems, what is the role of gluons and whether quark-gluon configurations other than baryons and mesons exist.

In this field a key tool is given by meson spectroscopy. Meson, being made by a quark and an antiquark, is the simplest quark bound system and therefore the ideal benchmark to study the interaction between quarks and understand what the role of gluons is. Historically, the study of meson properties led to some of the most relevant discoveries in particle physics. Some examples are the discovery of the pion by Powell, Occhialini and Lattes in 1947 [1] (see Fig. 1, left), the discovery of strange particles by Rochester and Butler in the same year [2] (see Fig. 1, right), the interpretation of the $\phi \rightarrow K K$ decay by Zweig and others in 1963 [3], the discovery of the $J / \phi$ in 1974 [4]. These findings were crucial to conjecture the existence of quarks of different flavors and put the grounds for the development of QCD.

\section{Meson spectroscopy in the light quark sector}

After many years, meson spectroscopy remains a very active field and a precious source of information for the understanding of quark-gluon interaction through a precise determination of the spectrum and properties of ordinary mesons $(q \bar{q})$ and the search of states with unconventional quark-gluon configuration. 


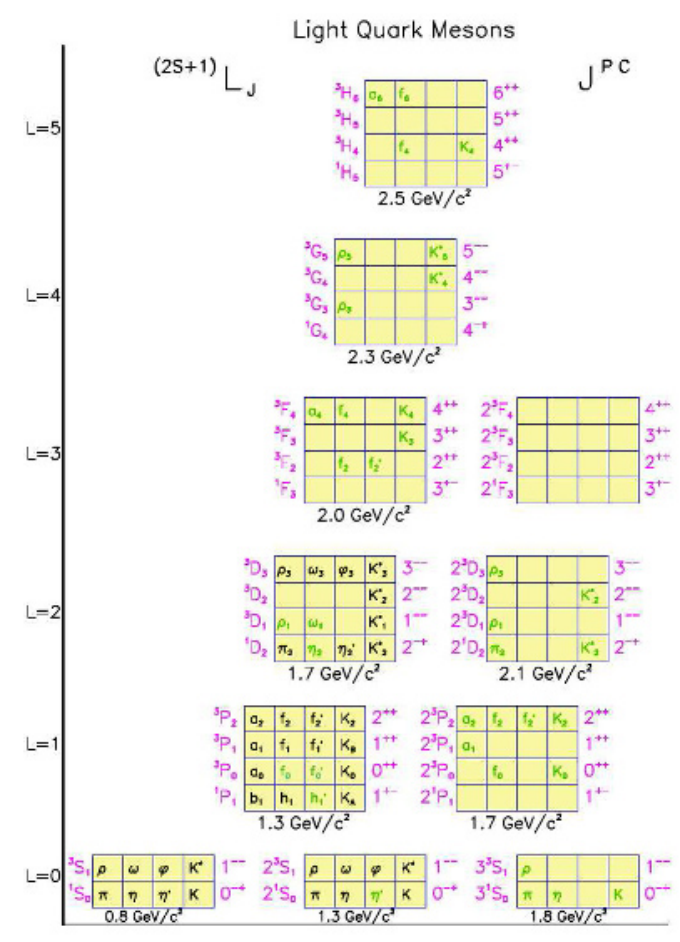

Figure 2. Quark model predictions of the meson spectrum in the light quark sector [6]. Each yellow box corresponds to a nonet of degenerate states predicted by the model. Names indicated in the boxes correspond to well-established states (black) and tentative assignments (green).

Experimental and theoretical studies of mesons composed of light quarks, i.e. $u, d$ and $s$, or heavy quarks, i.e. $c, b$ and $t$, have been exploited to investigate different regimes of quark-quark interaction. In these proceedings, I will focus on the sector of light quark mesons. These states have a fundamental role for the understanding of strong interaction since they are sensitive to chiral symmetry breaking and vacuum condensate effects. In addition, they allow us to probe the strong force at large distances where linear $q \bar{q}$ potential leading to confinement is expected to arise as indicated by Lattice QCD calculations [5].

The constituent quark model, where mesons are described as quark-antiquark pairs that couple to give total spin $S=0,1$ and have orbital angular momentum $L$, can provide first indications on the spectrum. Assuming SU(3) flavor symmetry to apply to the $u d s$ sector, nonets of degenerate states with the same quantum numbers, $J^{P C}$, are expected for each value of $L$ and $S^{1}$. The actual mass of the states depends on the mass that is assigned to the constituent quarks, typically few hundred $\mathrm{MeV}$, and on the $q \bar{q}$ potential that is chosen and that determines the eigenstates of the system. A typical prediction of the light-quark meson spectrum based on the constituent quark model approach is summarized in Fig. 2. In this diagram, each yellow box corresponds to a nonet of degenerate states with fixed $J^{P C}$ as a function of the radial excitation of the $q \bar{q}$ system on the horizontal axis and of the orbital angular momentum on the vertical axis. Names in the boxes indicate well-established states (black) and

\footnotetext{
${ }^{1}$ In this notation, $J$ is the total angular momentum, $P$ is the parity and $C$ the charge conjugation of the $q \bar{q}$ pair.
} 


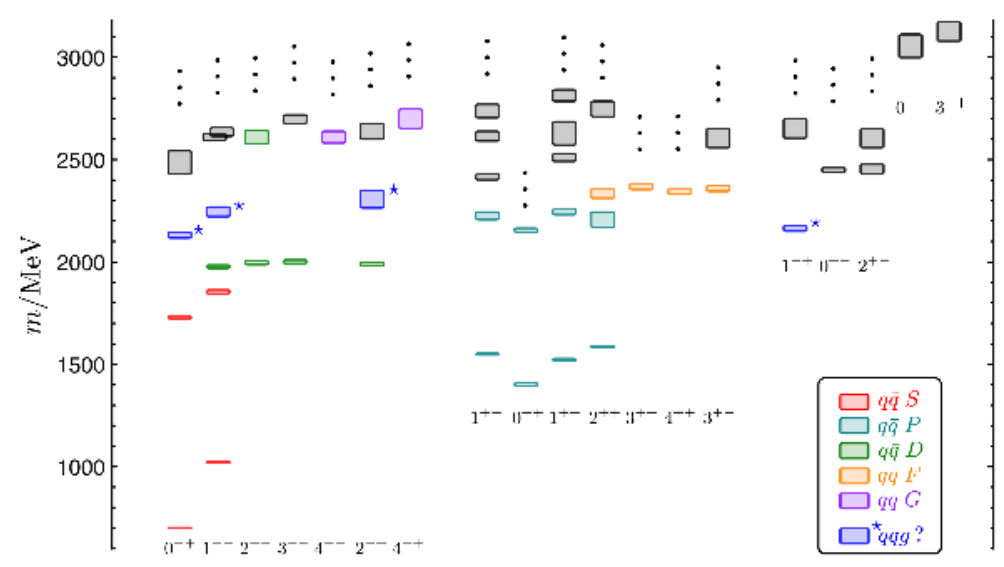

Figure 3. Spectrum of isovector mesons from Lattice QCD calculations with a pion mass of $700 \mathrm{MeV}$. Boxes indicate the predicted states as a function of the quantum numbers $J^{P C}$. In addition to states with quantum numbers that are consistent with the quark-model expectations for $q \bar{q}$ states, states with exotic quantum numbers are also predicted. The plot is from Ref. [7].

tentative assignments (green). While the quark model is very successful in describing the low-lying states, at higher masses a number of predicted states is not experimentally observed and assignments are often only tentative. In the last decades, this issue has been the subject of many speculations and studies, but whether this indicates a major failure of the assumptions on which the quark model is built or is due to a lack of the experimental techniques used to identify meson resonances has still to be resolved.

In addition to the study of the regular meson spectrum, a strong interest has arisen for the search of unconventional meson states, beyond the $q \bar{q}$ configuration. In fact, while QCD tells us that bound states have to be color neutral, it does not prohibit the existence of states with unconventional configurations such as tetraquarks ( $q q \bar{q} \bar{q})$, molecules $(q \bar{q}-q \bar{q})$, hybrids $(q \bar{q} g)$ and glueballs. These states can be distinguished unambiguously from regular mesons when they have quantum numbers (i.e. combinations of total angular momentum, spin and parity) that are not allowed for $q \bar{q}$ states. These are called exotic quantum numbers and the corresponding states are referred to as exotics. Existence of these states is supported by several phenomenological models and, recently, also by Lattice QCD. Recent calculations [7] based on a very large operator basis were used to predict the spectrum of isovector and isoscalar mesons. The results reproduce well the pattern and masses of the regular meson spectrum but also provide indications of the existence of states with exotic quantum numbers, as shown in the diagram in Fig. 3. Studying the overlap of these states with operators involving both quark and gluon fields, the authors were able to identify these multiplets as candidates for hybrid mesons, providing a very important indication to guide experimental searches.

Evidence for unconventional states has been searched in many laboratories. As an example, the $\mathrm{X}(3872)$ resonance recently observed at B-factories [8] has been suggested as a candidate for a tetraquark or a molecular state. Indications of resonances with exotic quantum numbers and masses below $2 \mathrm{GeV}$ were reported by several experiments [9]. While these findings are not yet sufficient to claim a discovery, these states, if unambiguously confirmed, would provide the mean to further investigate aspects of QCD as confinement and gluonic excitations. 

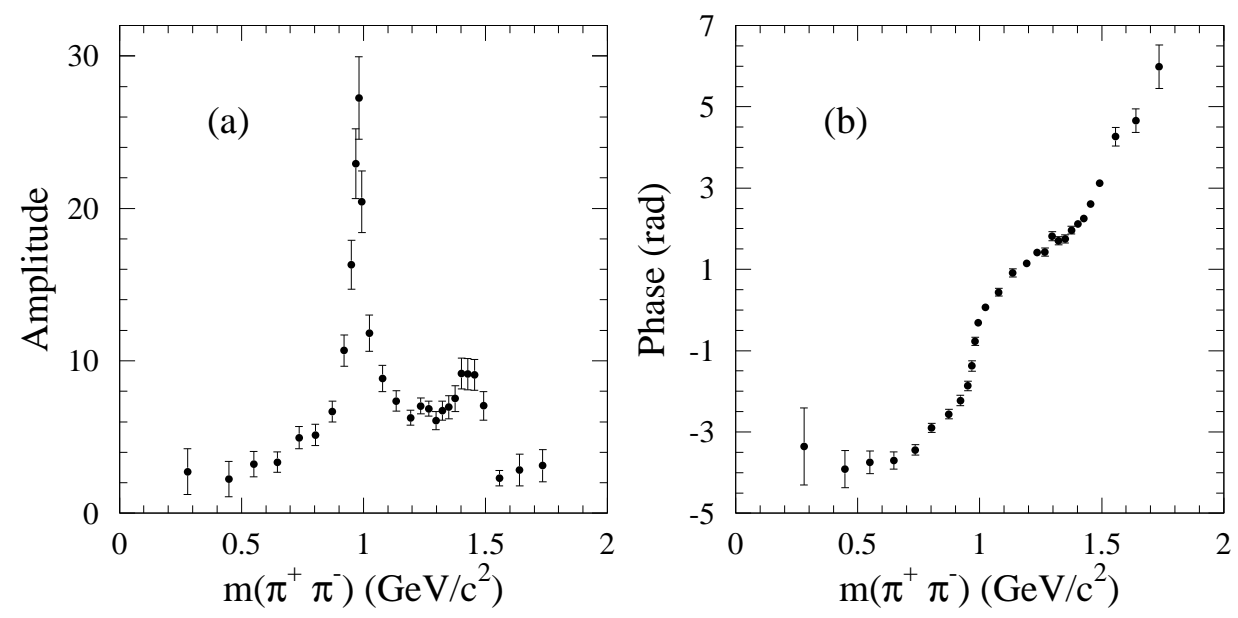

Figure 4. S-wave amplitude (left) and phase (right) extracted from the analysis of $D_{S}^{+} \rightarrow \pi^{+} \pi^{-} \pi^{+}$data from the Babar experiment [11].

\section{Experimental Studies}

The importance of the physics that can be accessed via meson spectroscopy studies has motivated many laboratories in the world to launch extensive experimental programs to investigate the meson spectrum in the light-quark sector using different production processes. These include protonantiproton annihilation, $e^{+} e^{-}$annihilation, proton-proton scattering, pion scattering on fixed target and photo-production experiments. The use of different probes is crucial in this field since the production mechanisms of meson resonances are often unknown and different reactions can provide complementary sensitivity.

This broad experimental activity has led to a very rich phenomenology: in the following I will discuss recent results in some of the most active sectors.

\subsection{Scalar mesons}

Scalar mesons with $J^{P C}=0^{++}$are some of the most intriguing bound states in QCD. These states have a fundamental role for strong interaction, being the equivalent of the Higgs boson. They have in fact the same quantum numbers of the QCD vacuum and the lowest mass state, the so-called $f_{0}(600)$ or $\sigma$, is the field that in the non-linear $\sigma$ model breaks chiral symmetry giving mass to the goldstone bosons of QCD. The scalar sector presents however some of the most intriguing puzzles because, even if several states have been identified, the interpretation of their structure in terms of constituents is still quite unclear and assignments in the quark model classification are at most tentative. Open issues involve both the lowest lying states that should form the first nonet and the higher mass states. For masses below $2 \mathrm{GeV}$, there are two $\mathrm{I}=1$ and two $\mathrm{I}=1 / 2$ states that are reasonably well identified and could be part of two nonets. In the same mass range five $\mathrm{I}=0$ states have been observed, i.e. one more than what would be needed to complete the two nonets. This excess of resonances has led to speculations about the possible presence of an unconventional state, a glueball or a tetraquark. In particular, the unusual mass hierarchy of the first multiplet where the isoscalar $f_{0}(980)$ and isovector $a_{0}(980)$ are almost degenerate, has led to speculations about the possibility of these states being 

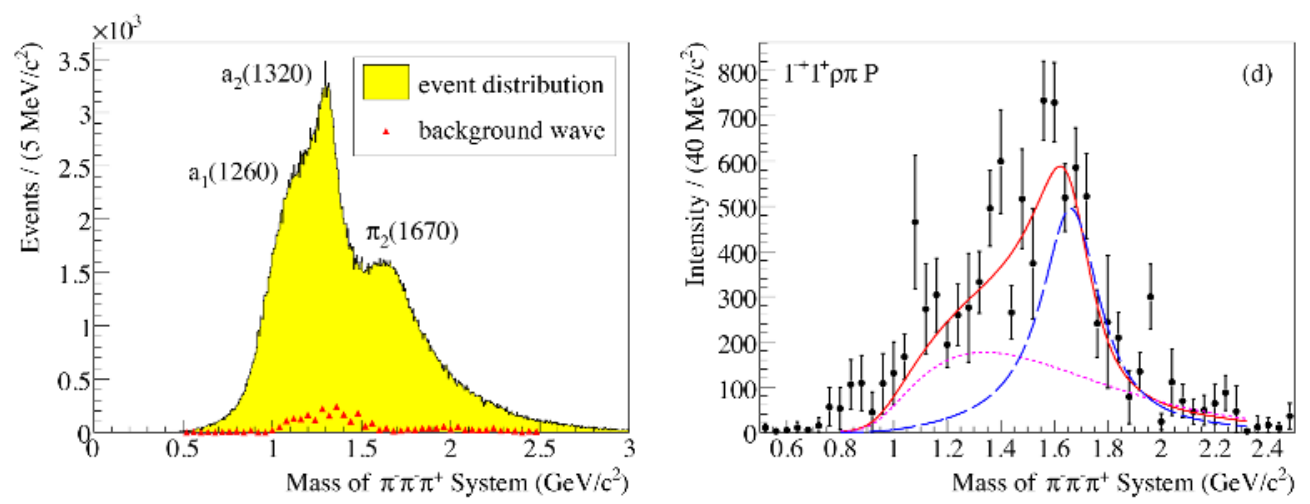

Figure 5. Results of the analysis of the $3 \pi$ final state from the 2004 pilot run of the COMPASS experiment [17]. Left: $3 \pi$ invariant mass spectrum showing clear evidence of structures corresponding to known resonances. Right: exotic $1^{-+}$wave extracted via a full partial wave analysis of the data set; the structure at $1600 \mathrm{MeV}$ was interpreted as evidence for the $\pi_{1}(1600)$.

tetraquarks instead of regular $q \bar{q}$ states. Testing this hypothesis and determining the real nature of these resonances requires high precision data for the scalar wave amplitude to allow an accurate extraction of the resonance poles. As an example, Fig. 4 shows some recent results obtained from the analysis of Babar data [11]. The very high statistics and accuracy of this and other data samples (see for example [12-14]) allow the use of very fine energy binning and sophisticated analyses that will hopefully provide decisive information about the structure of scalar resonances in the near future.

\subsection{Search for hybrid states}

The strong interest in the search for hybrid mesons is motivated by the fact that these states are the ideal benchmark to study quark-quark interaction and excitations of the glue. As mentioned before, the existence of hybrid states is supported by several theoretical models and by lattice QCD calculations that indicate that the lightest states could have masses below $2 \mathrm{GeV}$ and exotic quantum numbers $J^{P C}=1^{-+}$.

Experimentally the most debated evidence is related to the so-called $\pi_{1}(1600)$ that, if confirmed, would have indeed the quantum numbers and mass suggested by lattice QCD. Observation of this resonance have been reported by different experiments (E852[15], VES[16], COMPASS [17]) in several decay modes as $\rho \pi, \eta^{\prime} \pi, f_{1} \pi$ and $b_{1} \pi$. These include decays to meson pairs with orbital angular momentum $\mathrm{L}=1$ and $\mathrm{L}=0$, as predicted by the flux tube model [10]. However some inconsistencies in the observed production mechanisms and negative results reported by other Collaborations [18, 19] demand for additional confirmations. One of the most recent analysis is from the COMPASS experiment at the CERN SPS, where scattering of a $192 \mathrm{GeV}$ pion beam on nuclear targets is used to produce multi-particle final states. A first positive observation was reported in Ref. [17] from the analysis of the $3 \pi$ final states from the 2004 pilot run, where a significant strength in the exotic $1^{-+}$wave was found at masses of about $1600 \mathrm{MeV}$ (see Fig. 5). While the accuracy of these results is not yet enough to claim the discovery, the analysis of the full data set is in progress and new results are expected. 

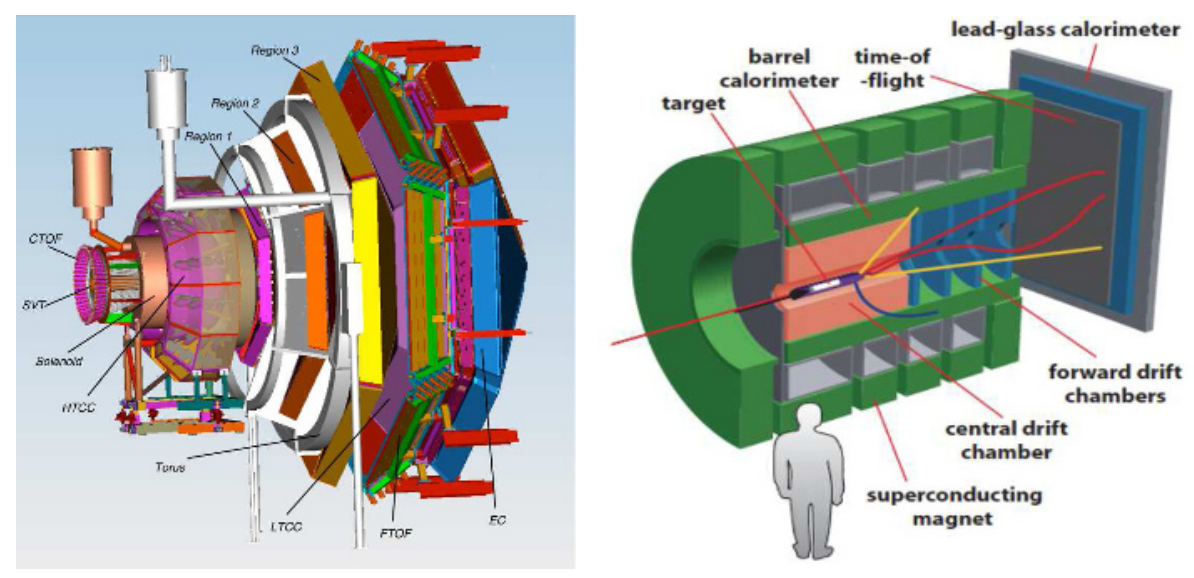

Figure 6. Schematics of the CLAS12 (left) and GlueX (right) experiments at Jefferson Lab.

\section{Future perspectives}

While existing data sets are still being analyzed, new experiments are also being designed to start data taking in the next few years and provide further insights in this field. Among the new facilities that have not yet started their activity, the PANDA experiment at GSI and the Belle II experiment at SuperKEKB will mainly focus on the heavy quark sector. On the contrary, new experiments at Jefferson Lab will focus on the light quark sector and are expected to be among the primary actors in this field. These are the CLAS12 [20] and GlueX [21] experiments that are presently being installed in the Hall B and Hall D of Jefferson Lab, as part of the $12 \mathrm{GeV}$ upgrade of the laboratory. Jefferson Lab hosts the Continuous Electron Beam Accelerator Facility (CEBAF), which is an electron machine based on two Linacs in racetrack configuration with superconducting cavities operating at high frequency. The electron beam which is produced is almost continuous and therefore ideal for the study of multi-particle final states. The ongoing upgrade will lead to an increase of the maximum beam energy from 6 to $12 \mathrm{GeV}$. Both CLAS12 and GlueX will investigate the meson spectrum and search for exotics, studying multi-particle final states produced by photo-production reactions. GlueX will use a linearly polarized tagged photon beam produced from the primary electron beam by coherent Bremsstrahlung on a diamond radiator, with a photon flux of about $10^{8} \mathrm{\gamma} / \mathrm{s}$. The detector (see the left panel of Fig. 6) is based on a solenoidal magnet and provides an almost complete coverage with flat and uniform acceptance, both for neutral and charge particles, thanks to the combined use of trackers, time of flight detectors and calorimeters. CLAS12 will use a complementary technique by studying meson resonances produced by low $Q^{2}$ electron scattering of the primary beam on a hydrogen target. In this kinematics, the virtual photons producing the final hadronic state can be considered as quasi-real and their energy and polarization degree are determined by detecting the scattered electron at small angle in a dedicated detection system called Forward Tagger [22]. The high resolution and good particle identification capabilities of the CLAS12 spectrometer will provide a detailed mapping of the meson spectrum up to masses of $\sim 2.5 \mathrm{GeV}$, with specific focus on the study of strangeonium and strangeness-rich states and search for exotics. 


\section{Summary}

Meson spectroscopy is a key field for the understanding of open questions in hadronic physics as what is the origin of the nucleon mass and what is the role of gluons. Primary focus of the research activity in this field is to establish the quark-antiquark bound state spectrum and search for exotic configurations. Experiments all over the world are collecting high statistics and high precision data, providing new insight in the meson spectrum. The new experimental data supported by recent theoretical developments can lead physicists to solve some of the most intriguing puzzles in hadronic physics.

\section{References}

[1] C. F. Powell, The cosmic radiation, Nobel Lecture, December 11, 1950.

[2] G.D. Rochester and C.C. Butler, Nature 160855 (1947).

[3] G. Zweig, The origin of the quark model, Talk at Baryons 1980 Conference, CALT-68-805.

[4] J. J. Aubert et al., Phys. Rev. Lett. 33, 1404 (1974); J. E. Augustin et al., Phys. Rev. Lett. 33, 1406 (1974).

[5] S. Godfrey and J. Napolitano, Rev. Mod. Phys. 71, 1411 (1999).

[6] C. Meyer, Lectures presented at the 1999 HUGS summer school.

[7] J. Dudek et al., Phys. Rev. D84, 074023 (2011) and references therein.

[8] R. Aaji et al [LHCb Collaboration], Phys. Rev. Lett. 110, 222001 (2013)

[9] See for example C. A. Meyer and Y. Van Haarlem, Phys. Rev. C 82, 025208 (2010), V. Crede and C. A. Meyer, Prog. Part. Nucl. Phys. 63, 74 (2009) and references therein.

[10] P. R. Page, E. S. Swanson and A. P. Szczepaniak, Phys. Rev. D 59, 034016 (1999).

[11] B. Aubert et al, Phys. Rev D 79, 032003 (2009).

[12] M. Ablikim et al. [BESIII Collaboration], Phys. Rev. D. 87, 092009 (2013).

[13] F. Ambrosino et al. [KLOE Collaboration], Eur. Phys. J. C 49, 473 (2007).

[14] M. Battaglieri et al. [CLAS Collaboration], Phys. Rev. Lett. 102, 102001 (2009); M. Battaglieri et al. [CLAS Collaboration], Phys. Rev. D 80, 072005 (2009).

[15] G. S. Adams et al. [E852 Collaboration], Phys. Rev. Lett. 81, 5760 (1998); S. U. Chung et al., Phys. Rev. D 65, 072001 (2002); J. Kuhn et al. [E852 Collaboration], Phys. Lett. B 595, 109 (2004).

[16] Y. P. Gouz et al. [VES. Collaboration], AIP Conf. Proc. 272, 572 (1993); Y. A. Khokhlov [VES Collaboration], Nucl. Phys. A 663, 596 (2000); A. Zaitsev [VES Collaboration], Nucl. Phys. A 675, 155C (2000).

[17] M. Alekseev et al. [COMPASS Collaboration], Phys. Rev. Lett. 104, 241803 (2010).

[18] A. R. Dzierba et al., Phys. Rev. D 73, 072001 (2006).

[19] M. Nozar et al. [CLAS Collaboration], Phys. Rev. Lett. 102, 102002 (2009).

[20] Jefferson Lab Experiment E12-11-005, M. Battaglieri et al, Meson spectroscopy with low $Q^{2}$ electron scattering in CLAS12.

[21] Jefferson Lab Experiment E12-06-102, C. Meyer et al., Mapping the Spectrum of Light Quark Mesons and Gluonic Excitations with Linearly Polarized Photons.

[22] M. Battaglieri et al. [CLAS Collaboration], The CLAS12 Forward Tagget (FT) Technical Design Report, http://www.ge.infn.it/ batta/jlab/ft-tdr.2.0.pdf. 